\title{
Explicit- and Implicit Bullying Attitudes in Relation to Bullying Behavior
}

\author{
Anne A. J. van Goethem • Ron H. J. Scholte • \\ Reinout W. Wiers
}

Published online: 30 March 2010

(C) Springer Science+Business Media, LLC 2010

\begin{abstract}
The main aim of this study was to examine whether an assessment of implicit bullying attitudes could add to the prediction of bullying behavior after controlling for explicit bullying attitudes. Primary school children (112 boys and 125 girls, $M$ age=11 years, 5 months) completed two newly developed measures of implicit bullying attitudes (a general Implicit Association Test on bullying and a movie-primed specific IAT on bullying), an explicit bullying attitude measure, and self reported, peer reported, and teacher rated bullying behavior. While explicit bullying attitudes predicted bullying behavior, implicit attitudes did not. However, a significant interaction between implicit and explicit bullying attitudes indicated that in children with relatively positive explicit attitudes, implicit bullying attitudes were important predictors of bullying behavior. Theoretical and practical implications are discussed.
\end{abstract}

Keywords Explicit bullying attitudes .

Implicit bullying attitudes $\cdot$ Interaction explicit and implicit bullying attitudes $\cdot$ Bullying

During the research project on implicit and explicit bullying attitudes all authors were working at the Behavioural Science Institute, Radboud University, Nijmegen, The Netherlands

A. A. J. van Goethem $(\bowtie)$

Institute of Developmental Psychology, University of Utrecht, P.O. Box 80140, 3584 CS Utrecht, The Netherlands

e-mail: A.A.J.vanGoethem@uu.nl

R. H. J. Scholte

Behavioural Science Institute, Radboud University,

Nijmegen, The Netherlands

R. W. Wiers

Developmental Psychology, University of Amsterdam, Amsterdam, The Netherlands
School bullying is a large societal problem and is already evident among primary school children. Studies on this population show that $3-27 \%$ of the children bully, and 9$32 \%$ are bullied once a week or more (Berger 2007). Bullying has also been shown to have large negative consequences for the well-being of victims and bullies (Berger 2007; Scholte et al. 2007), which stresses the importance of targeting bullying behavior together with the factors that are related to bullying.

Various factors have been found to contribute to the extent to which children bully other children. Research evidence indicates that social factors such as group membership and peer pressure, as well as individual, personal factors such as physical strength, aggressiveness, and empathy influence bullying (Rigby 2004). Another often studied individual factor is children's attitudes towards bullying. Attitudes are predictors of all kinds of spontaneous and deliberate social and non-social behavior (e.g., Glasman and Albarracin 2006) including bullying behavior (Salmivalli and Voeten 2004). Attitudes can be defined as general and enduring, concrete or abstract evaluations of a person, group, or issue and can be based on beliefs, emotions and behavior (cf. Petty and Cacioppo 1986).

In attitude research, a distinction is made between implicit attitudes and explicit attitudes. Implicit attitudes are impulsive, spontaneous, uncontrolled emotional reactions and evaluations. In contrast, explicit attitudes refer to deliberate, reflective, controlled, consciously self-reported evaluations (Gawronski and Bodenhausen 2006). Dualprocess models assume that there are two different modes of information processing that underlie implicit and explicit attitudes. According to these models more automatic, impulsive, associative processes underlie implicit attitudes and more controlled, reflective processes underlie explicit 
attitudes. These models also assume that behavior is caused by the interplay of these two kinds of processes and attitudes (Evans 2008; Gawronski and Bodenhausen 2006; Smith and DeCoster 2000). Dual-process models have gained wide support in various areas of psychology, including research on aggression (e.g., Hofmann et al. 2008).

The different nature of explicit versus implicit attitudes is also translated into the way the two concepts are measured. Explicit attitudes are measured directly by using self-reports (e.g., questionnaires): participants consciously express what they feel or think about a certain topic or subject. Implicit attitudes are measured differently. As self-reports are controlled evaluations, they are not suited to measure implicit attitudes, which are uncontrolled evaluations. Instead, implicit attitudes are indirectly inferred from participants' (impulsive) reaction times to stimuli (e.g., Implicit Association Test).

The distinction between implicit and explicit attitudes can also be applied to the field of bullying research: implicit bullying attitudes are impulsive, uncontrolled feelings towards bullying in general (e.g., attitudes towards bullying behavior, or towards a person who bullies). These attitudes can be distinguished from explicit bullying attitudes which are deliberate and controlled feelings or thoughts towards bullying in general (e.g., attitudes towards bullying behavior, or towards a person who bullies). For example, when a teacher shows pupils a movie in which a child is bullying another child, it could be that a pupil in the class who is a bully will have an initial, immediate, positive appraisal of bullying while watching this movie. This is what we refer to as a positive implicit attitude towards bullying or an implicit probullying attitude. However, when the teacher asks what the students think of the movie, it could be that the pupil who is a bully deliberately expresses disapproval of bullying, after realizing that bullying is disapproved of, and probably punished by most teachers. This is what we refer to as a negative explicit attitude towards bullying, or an explicit antibullying attitude. Furthermore, as in other attitude research, implicit bullying attitudes can be measured using an indirect measure such as the Implicit Association Test (IAT; Greenwald et al. 1998) and explicit bullying attitudes can be measured directly using self-report questionnaires.

Strikingly, until now bullying research and programs have only focussed on explicit bullying attitudes (these attitudes are most often assessed on likert-scales, asking children to what extent they agree with various statements on bullying; Boulton et al. 2002; Menesini et al. 1997; Salmivalli and Voeten 2004) and their relation to bullying behavior (see for a review Stevens et al. 2000). These studies show that the majority of school-aged children have a negative explicit bullying attitude whereas only a minority have a positive explicit bullying attitude (e.g. Andreou et al. 2005; Menesini et al. 2003). Furthermore, children's explicit bullying attitudes are also related to their bullying behavior. Generally, children with more negative explicit bullying attitudes bully less than children with more positive explicit bullying attitudes. Although this is the case, research has shown that explicit bullying attitudes of children are only minor to moderate predictors of bullying behavior (e.g., Boulton et al. 2002; Rigby 2004; Salmivalli and Voeten 2004).

The finding that explicit bullying attitudes have low to moderate predictive power for bullying behavior could indicate that children's explicit bullying attitudes are not always in accordance with their bullying behavior: some children may report in questionnaires that they have negative explicit bullying attitudes but may still show bullying behavior. This paradox was also raised by Salmivalli and Voeten (2004) who indicated that although the majority of children disapprove of bullying, a lot of children and adolescents are directly or indirectly involved in bullying. The question then arises whether another factor could add to the prediction of bullying behavior. We expect that implicit bullying attitudes could be an additional factor explaining bullying given their relevance for related behaviors, such as aggression (Gollwitzer et al. 2007; Hofmann et al. 2008; Wiers et al. 2009). Furthermore, as bullying is considered to be socially unacceptable behavior, explicit bullying attitudes could be more influenced or controlled by factors such socially desirable answering (Nosek 2005) than implicit bullying attitudes. Consequently, it could be the case that although children explicitly reject bullying in questionnaires, they have more relaxed implicit attitudes towards bullying which are more in accordance with their bullying behavior. Given the hypothesized relevance of implicit bullying attitudes in bullying behavior, the purpose of this study is to examine the role of implicit next to explicit bullying attitudes in explaining bullying behavior.

Up to now no research has been done on implicit bullying attitudes in relation to bullying. However, there is some indication of this relation from studies in other research areas which have shown that implicit attitudes are directly related to many kinds of behavior (Gawronski and Bodenhausen 2006; Nosek 2005) including socially disapproved behavior such as aggression (e.g., Gollwitzer et al. 2007). The predictive value of implicit (self-related) aggression attitudes in relation to aggressive behavior was found in a study of Gollwitzer et al. (2007). They found that the extent to which children implicitly thought of themselves as being aggressive was related to their actual aggressive behavior. Children who associated themselves more with aggression were more likely to show aggressive behavior than children who associated themselves less with aggression. As bullying is a form of aggression (Sullivan 2000), it is possible that a similar relation exists between bullying behavior and implicit bullying attitudes indicating that children with relatively more negative implicit bullying 
attitudes (implicit anti-bullying attitudes) will show less bullying behavior than children with more positive implicit bullying attitudes (implicit pro-bullying attitudes).

In addition to direct relations of both explicit and implicit bullying attitudes to bullying, explicit bullying attitudes could interact with implicit bullying attitudes in relation to bullying. As discussed, according to dual process models both automatic processes which underlie implicit attitudes, and more controlled processes which underlie explicit attitudes cause behavior (Evans 2008; Gawronski and Bodenhausen 2006; Kahneman 2003; Smith and DeCoster 2000). Both processes can influence behavior independently (Nosek et al. 2005). However, these attitudes and underlying processes are often related to each other (Nosek 2005) which could mean that they also influence each others' effects on behavior.

The interaction of implicit-and explicit attitudes and their underlying processes has recently attracted attention (see also Grenard et al. 2008; Thush et al. 2008) in the prediction of a variety of behaviors including aggression (Schröder-Abé et al. 2007a, b). For example, in a study on interracial interactions it was found that for low levels of inhibition, implicit racial attitudes predicted interracial interactions and for high levels of inhibition, explicit racial attitudes predicted these interactions (e.g., the competitiveness of the confederate reported by the participants, and the talkativeness of the participant; Hofmann et al. 2008). Executive control processes, including inhibition, are part of the reflective system, which also includes explicit attitudes (Hofmann et al. 2008). Under low executive control, reflective processing breaks down and impulsive processes, including implicit attitudes "take over" and predict behavior (e.g., Hofmann et al. 2008; Wiers et al. 2009). In the latter study for example, implicit associations relating alcohol to power predicted aggressive behavior after drinking alcohol in participants with relatively low executive control. In contrast, in participants with high executive control, implicit alcohol-power associations were not related to aggression after drinking alcohol. Similarly, implicit and explicit bullying attitudes could moderate each others' effects on bullying behavior.

We propose that the strength of the explicit bullying attitude could moderate the relation of implicit bullying attitudes to bullying behavior. For example, as inhibition underlies deliberate, controlled process, it could be that an extreme negative explicit bullying attitude is related to a strong motivation to inhibit implicit bullying attitudes. As found in aggression related research (Hofmann et al. 2008; Wiers et al. 2009), this strong inhibition would control implicit bullying attitudes and therefore weaken the influence of these implicit bullying attitudes on bullying behavior. For less extreme values of the explicit bullying attitudes, it could be that the amount of inhibition is low in which case implicit bullying attitudes, in addition to explicit bullying attitudes, could predict bullying behavior. If supported, this could be a working mechanism underlying effective bullying interventions which promote clear (explicit) anti-bullying rules (e.g., Olweus 1993).

\section{The Present Study}

In the present study we examined whether an assessment of implicit bullying attitudes could predict bullying behavior in addition to explicit bullying attitudes. In accordance with prior research we hypothesized that most children show negative explicit attitudes towards bullying and that explicit bullying attitudes are related to bullying. We also expected that implicit bullying attitudes are related to bullying. To test implicit bullying attitudes we used the Implicit Association Test (IAT; Greenwald et al. 1998).

The IAT is an often used instrument to assess implicit attitudes, including in bullying related research. For example, Gollwitzer et al. (2007) used an IAT to assess implicit (selfrelated) aggression attitudes and found a relation between these attitudes and aggressive behavior. In this study we used two newly developed varieties of the IAT: a general IAT on bullying (similar to self-concept IATs, e.g., Asendorpf et al. 2002) and a movie-primed IAT on bullying. A prime is a stimulus presented to participants preceding a test. Priming is a means through which external sources (e.g., a movie) automatically activate relevant evaluative associations in memory, such as implicit attitudes. After the prime has been presented, implicit attitudes are assessed with a succeeding test, such as an IAT (Perugini and Prestwich 2007). The bullying movie used here was expected to make the bullying behavior more concrete and was expected to further activate relevant bullying concepts and associations that were assessed by the IAT (a priming effect on implicit attitudes has also been shown in other domains, for example in alcohol research using an alcohol prime, Schoenmakers et al. 2008 or in research on suicidal cognitions using a video with suicidal and non-suicidal stimuli as a prime, Rustad et al. 2003), which could possibly lead to a stronger representation of implicit bullying attitudes measured by the IAT. We used these two different IATs, to investigate whether each IAT, a general implicit measure of bullying attitudes and a movieprimed implicit measure of bullying attitudes, could predict bullying behavior.

\section{Method}

Participants

Participants were 240 fifth grade $(n=115)$ and sixth grade $(n=122)$ children (112 boys and 125 girls) in 11 classes 
representing five regular primary schools. All schools had some form of anti-bullying policy. Children were between 10 and 13 years old $(\mathrm{M}=11.41$ years, $\mathrm{SD}=0.71)$. There were no significant relations between the age of the children and the bullying behavior (self-reports: $p=0.35$; teacherratings: $p=.021$; peer nominations: $p=0.60$ ) or attitude measures (the movie-primed IAT: $p=.98$; the general IAT: $p=$ 0.17 ; the explicit bullying attitude measure: $p=0.29$ ). Therefore the participants were treated as one age-group.

SES and ethnic background was recorded. The latter showing that $98 \%$ of the participants were of Dutch origin, while $2 \%$ of the children were ethnic minorities. Overall, the participants were representative of the Dutch school population in the geographic area in which the children lived. The participation rate was $99 \%$, and none of the participants refused nor did any parents forbid their children's participation in the study.

\section{Procedure}

After IRB approval for our study was acquired, school authorities were approached and asked to participate in our study. After these authorities had given their permission, schools sent letters to the parents of the children informing them about the study. Passive consent was obtained from parents and children. The data collection was performed in the winter of 2008 and contained two parts for each school class: a classroom session of $45 \mathrm{~min}$ and computer sessions within small groups. For practical reasons, classroom sessions preceded computer sessions (this has shown to have no significant effects on task-performance, Nosek et al. 2005).

Each classroom session started with a brief introduction and class instruction, given by a trained examiner. In this instruction children were guaranteed confidentiality in the collection and maintenance of the data and were told they were free to ask questions and to skip personally sensitive questions. Furthermore, children were instructed to use student numbers to identify themselves and their classmates. Teachers were also instructed to use student numbers to identify their students.

During the assessment, children and the teacher were asked to fill out a questionnaire which contained the bullying behavior measures. For children, the questionnaire also included the explicit bullying attitude scale. During the computer-sessions, children were given short instructions by a trained examiner who also supervised the children as they worked individually on two versions of the Implicit Association Test (IAT) that measured their implicit bullying attitudes. These IATs were counterbalanced to control for the possibility that practice with IAT during the assessment of the first IAT measure, would influence the performance on the second IAT measure.
Measures

Bullying In the questionnaire we defined the concept "bullying" as follows:

We can speak of bullying when a child or a group of peers says malicious or hurtful things to another child or laughs at another child. The same is true when a child is being hit, kicked, threatened, or properties are being taken away or destroyed. Furthermore, it is bullying when a child is being excluded from the group, or when other children gossip about the victim. These things can be classified as bullying when they happen frequently or regularly, and when it is difficult for the child being bullied to defend him- or herself. It is not bullying when two or more children who are equally strong tease each other or fight with each other (cf. Olweus 1993).

We used three measures to assess bullying, each containing one bullying-item (it has been shown that bullying can be reliably assessed using one item for bullying behavior, Solberg and Olweus 2003).

(1) Self-reports: one item of the Olweus Bully/Victim self-report questionnaire which measured for each child how many times the child bullied other children in their class this school year. Answers were made on a five-point scale, ranging from "never" to "several times a week". (2) Peer nominations: each child nominated classmates who bullied in their class. The number of nominations was unlimited and both same-sex as opposite-sex nominations were allowed. The statistical program SocStat (Thissen and Bendermacher 1996) was used to calculate children's score on the bullying item. For each item, the program summed the number of nominations received by each child and transformed them into z-standardized scores. In the calculation of these scores, the program took into account how many nominations a student could maximally receive given the class size (Newcomb and Bukowski 1983). (3) Teacher ratings: teachers rated for each child how many times the child bullied other children in their class this school year on a five-point scale, ranging from "never" to "several times a week" (Olweus 1989).

Explicit Bullying Attitudes A Dutch translation of the bullying attitude scale by Salmivalli and Voeten (2004) was used. This is an often applied measure of explicit bullying attitudes and has satisfactory predictive validity as it is found to be associated with bullying behavior (Salmivalli and Voeten 2004). Salmivalli and Voeten (2004) reported this scale has satisfactory internal consistency, with Cronbach's coefficient alpha of 0.75 . In our study, we found a similar internal consistency of $\alpha=0.76$. Children were asked to evaluate on a 5-point scale, (1 
strongly disagree, 5 strongly agree) the extent to which they agreed or disagreed with 10 statements about bullying. These statements were, for example, "Bullying may be fun sometimes", "It is not that bad if you laugh with others when someone is being bullied", and "Joining in bullying is a wrong thing to do" (this statement was reversed coded). An attitude score was formed by averaging children's scores on all statements. A low average score indicated a more negative explicit bullying attitude (i.e., bullying is bad, anti-bullying), whereas a high average score indicated a more positive explicit bullying attitude (i.e., bullying is good, pro-bullying).

Implicit Bullying Attitudes Implicit bullying attitudes were assessed with two versions of the Implicit Association Test (IAT): a general IAT on bullying (general IAT) and a movie-primed specific IAT on bullying (specific IAT). The IAT is a reliable and valid measure of implicit attitudes (Greenwald et al. 2006; Nosek et al. 2005) and has been used with children (Baron and Banaji 2006). The IAT is a double discrimination task in which participants are asked to assign single stimuli as fast as possible to a given pair of target categories and attribute categories using two response keys. A pair of target categories is combined with a pair of attribute categories in an association-congruent and an association-incongruent manner. In our study, the attribute categories "pleasant" versus "unpleasant" were combined with the target categories "bullying" versus "non-bullying". Pleasant and non-bullying sharing one response key, and unpleasant and bullying sharing another response key was the congruent association. Pleasant and bullying sharing one response key, and unpleasant and non-bullying sharing another response key was the incongruent association. The difference between the reaction times for associationcongruent and association-incongruent assignments is usually interpreted as an indicator of the IAT effect: the relative associative strength between the two pairs of concepts. The underlying assumption is that when two categories (e.g., unpleasant and bullying) are more strongly associated in memory, responses are faster when they share the same response-key. In our study for example, how fast participants are to sort pictures of bullying together with positive as compared to negative stimuli, would be interpreted as their implicit attitude toward bullying. The D-2SD penalty score for practice and test was chosen as the main reaction time measure, which can roughly be interpreted as an individualized effect size (.5 medium effect, 0.8 large effect, Greenwald et al. 2003). D measures, which are based on all trials of the combination blocks (including the practice phases), give an error-penalty to the reaction times on erroneous responses, and divide the outcome with a personalized standard deviation (SD) of the combination phases. We used the D-2SD measure because it came out among the best algorithms in the study by Greenwald et al. (2003), and because children may have larger SDs than the standard $300 \mathrm{~ms}$ (the general estimate in the other often used algorithm, the D600 measure, cf. Thush et al. 2008).

The IATs were programmed in Inquisit version 2 (www. millisecond.com). Six bullying pictures vs. six non-bullying pictures were used as target stimuli categorized into bullying vs. non-bullying. For both IATs, the bullying pictures showed various bullying interactions between children: relational, verbal and physical bullying. The non-bullying pictures showed children friendly laughing and, in some pictures, putting their arms around each other. The general IAT on bullying contained pictures that were unfamiliar to the participants. The movie-primed version of the IAT on bullying contained pictures derived from the movie on bullying preceding the IAT. For example, in one picture Don, a boy who is the leading character of the movie, is being grabbed and hit by a group of boys. This picture represents physical bullying. For the attribute stimuli, six pleasant words ("Love", "Sunshine", "Warmth", "Peace", "Hug", and "Rainbow") vs. six unpleasant words ("Distress", "War", "Bad", "Pain", "Death", "Disease") were used and categorized into "pleasant" vs. "unpleasant" (e.g., Houben and Wiers 2007). Children first practiced the attribute classification (pleasant vs. unpleasant words) with a right and a left response key (20 trials). In the second block, they practiced the target classification (bullying vs. non-bullying pictures) with the same response keys ( 20 trials). The third block was the first combination block during which both target stimuli (bullying and non-bullying pictures) and attribute stimuli (pleasant and unpleasant words) were combined. For example, when stimuli referred to pleasant words and non-bullying pictures, the left response key was pressed, and when stimuli referred to unpleasant words and bullying pictures the right key was pressed (40 trials: 20 trials practice and 20 trials test). In the next phase, the children practiced the target classification with reversed response keys (bullying and non-bullying pictures, 40 trials; the trials were doubled to reduce the overall impact of block order within the IAT, Nosek et al. 2005). This practice phase was followed by the reversed combination block. In this reversed combination block the left response key was for example used for pleasant words and bullying pictures and the right response key was used for unpleasant words and non-bullying pictures (40 trials). An overview of all IAT blocks is presented in Table 1.

Half of the participants received this IAT in which the compatible combination comes first (bullying with unpleasant and non-bullying with pleasant). The other half of participants first had the incompatible combination (bullying with pleasant and non-bullying with unpleasant). Furthermore, the response assignment of the target categories 
Table 1 Overview of IAT Blocks

The "Non-bullying" and "Bullying" stimuli are pictures and the "Pleasant" and "Unpleasant" stimuli are words; during reversed attribute practice, trials were doubled to reduce the overall impact of block order within the IAT (Nosek et al. 2005)

\begin{tabular}{lllll}
\hline Block & Trials & Function & Left key & Right key \\
\hline 1 & 20 & Attribute practice & Pleasant & Unpleasant \\
2 & 20 & Target practice & Non-bullying & Bullying \\
3 & 20 practice & Compatible combination & Pleasant & Unpleasant \\
& 20 test & & Non-bullying & Bullying \\
4 & 40 & Reversed attribute practice & Bullying & Non-bullying \\
5 & 20 practice & Incompatible combination & Pleasant & Unpleasant \\
& 20 test & Bullying & Non-bullying & \\
\hline
\end{tabular}

to the left and right response keys and the position of the attribute-words (pleasant-unpleasant) were counterbalanced. Instructions were presented before each task. During the task, the labels of the categories assigned to the left and right response key were presented in the corresponding upper corners of the computer screen. Target and attribute stimuli were presented randomly in an alternating order in the middle of the computer screen against a black background. All textual elements (attribute-and target labels and attribute stimuli) were presented in white. Stimuli disappeared when a response was given or after $5,000 \mathrm{~ms}$ (hereinafter the response can not be analyzed) when no response was given. The inter-trial interval was $250 \mathrm{~ms}$. Error feedback was presented in red beneath the stimuli after an incorrect response ("X"). We used it in order to check whether children correctly understood the experimental procedure. This check is especially important because the procedure subtly changes (the combination of the categories: positive-negative and bullying-non-bullying changes) per block of the IAT which increases the chance of a high error-rate. A high error rate consequently leads to a high percentage of data that can not be analyzed.

In a pilot study, different bullying and non-bullying pictures were pre-tested. Twenty-five fifth-grade children were asked to classify pictures as bullying pictures or nonbullying pictures. The pictures that were most often classified correctly were selected for our final version of both IATs. Both IATs are available upon request. The movie we used for the specific IAT was a 10-min compilation of fragments of bullying interactions, pleasant interactions and text fragments. As a whole it told a story about a boy, Don, who is initially being bullied by his classmates but is liked by all of them at the end of the movie. The movie-fragments were derived from a Dutch movie for children called "Don" which is also available upon request. The movie was expected to make the bullying behavior more concrete and was expected to further activate relevant bullying concepts and associations that were assessed by the IAT.

Internal consistencies were calculated for each IAT version by using the Spearman-Brown corrected correlation of the D measure calculated for the practice trials of the combination blocks with the IAT effect measure calculated for the test trials of the combination blocks (cf. Greenwald et al. 2003). Internal consistencies for the General IAT and the Specific IAT were 0.72 and 0.74 , respectively. In our study, a low average score indicated a more negative implicit bullying attitude (i.e. bullying is bad, anti-bullying), whereas a high average score indicated a more positive implicit bullying attitude (i.e., bullying is good, pro-bullying).

\section{Results}

For the IAT data, participants with error rates of more than $40 \%$ were removed. For the general IAT this concerned $2 \%$ of the participants, and for the movie-primed IAT this concerned $3 \%$ of the participants. No outliers among the IAT data were identified.

The descriptives of the variables are shown in Table 2. The z-standardized peer-nominations slightly differ from 1 due to the procedures used in the SocStat program. Overall, the average scores on bullying ratings were relatively low which indicates that bullying was reported as occurring infrequently. Explicit bullying attitudes were on average relatively low which indicates that most children had negative explicit bullying attitudes. The implicit attitude measures suggest that on average, children had negative implicit bullying attitudes.

The correlations between the various measures of bullying attitudes and bullying behavior are presented in Table 3. As shown, correlations between bullying behavior measures were moderate to high. The strongest relation existed between teacher ratings and peer nominations. Implicit bullying attitudes measured with the general version of the IAT were negatively related to implicit bullying attitudes measured with the specific version of the IAT. Explicit bullying attitudes were not related to implicit bullying attitudes.

The results of our regression analyses using the Complex Samples module in SPSS are presented in Table 4. The Complex Samples module allowed us to compute regression analyses while correcting the estimates, especially their standard errors and consequently also the significance tests, for effects of clustering by classrooms. Since children were 
Table 2 Mean Raw Scores, Standard Deviations and Scales for Bullying Behavior, Explicit Bullying Attitudes and Implicit Bullying Attitudes

\begin{tabular}{lllr}
\hline & $\mathrm{M}$ & $\mathrm{SD}$ & Scale \\
\hline Bullying behavior & 1.56 & 0.90 & $1-5$ \\
$\quad$ Self-reports & 1.42 & 0.75 & $1-5$ \\
$\begin{array}{l}\text { Teacher ratings } \\
\text { Peer nominations }\end{array}$ & 0 & 0.88 & $-4-4$ \\
$\begin{array}{l}\text { Bullying attitudes } \\
\text { Explicit attitudes }\end{array}$ & 1.80 & 0.50 & $1-5$ \\
$\begin{array}{l}\text { Implicit Attitude } \\
\text { (General IAT) }\end{array}$ & -0.97 & 0.57 & $-2-2$ \\
Implicit attitude $^{\mathrm{c}}$ & & & \\
$($ Specific IAT) & -1.03 & 0.58 & $-2-2$ \\
\hline
\end{tabular}

Descriptive statistics reported in this table concern the variables in their original metric

${ }^{a}$ The peer nominations are z-standardized

${ }^{\mathrm{b}}$ The implicit attitude measures are expressed in D-2SD reaction time scores on the two IATs we used

nested within classrooms the assumption of independently distributed residuals will be violated. Children within the same classroom tend to be more alike than children from different classrooms. This assumption was also tested by analyzing the homogeneity of the school classes with respect to the various measures of bullying behavior (dependent variable). The intra-class correlations (ICC) for these measures were larger than 0.05 which indicated that the effects of clustering on the regression analyses had to be considered.

To predict bullying behavior, explicit bullying attitudes, implicit bullying attitudes, the sex of the children, and the interaction between the explicit- and implicit bullying attitudes were entered in the regression. This procedure was applied in the analyses using self-reports, teacher ratings, and peer nominations of bullying behavior. For all analyses all variable scores were centred before entered in the regression analyses. The sex of the children was not related to bullying for self-reports but was related to bullying for teacher ratings and peer nominations, which indicated that boys bullied more than girls. Furthermore, as presented in both Tables 3 and 4, explicit bullying attitudes were moderately related to all measures of bullying, with relatively positive (high scores on) explicit bullying attitudes related to more bullying and relatively negative (low scores on) explicit bullying attitudes related to less bullying. We also found that implicit bullying attitudes were not related to bullying (this applied for the general and specific IAT).

However, as can be seen in Table 4, we did find a significant interaction between implicit bullying attitudes measured by the movie-primed IAT and explicit bullying attitudes in relation to bullying. This interaction was found for bullying according to teacher ratings and peer nominations but not for self-reports, and not for the implicit bullying attitudes measured with the general IAT. The effects of outliers on the interactions were checked using Dfbeta. Some Dfbeta's had an absolute value of $\left(2 / \sqrt{n}_{n}\right)$ or more. None of the cases with large Dfbeta's had a substantial influence on the significance of the parameter estimates of the interaction effect, except for one case. When this case was removed from the analyses, the interaction between implicit bullying attitudes and explicit bullying attitudes on bullying measured by teacher ratings became marginally significant $(p=0.06)$. However, this case had no influence on the significance of this interaction when bullying was measured with peer nominations. This model, including sex, explicit bullying attitudes, implicit bullying attitudes, and the interaction between implicit and explicit attitudes, explained $13 \%$ of the variance for the self-reports measure of bullying, $13 \%$ of the variance for the teacher ratings measure of bullying and $24 \%$ of the variance for the peer nominations of bullying.

The significant interactions between implicit bullying attitudes and explicit bullying attitudes are plotted in Figs. 1 and 2. Figure 1 shows individual teacher ratings of bullying as a function of children's implicit and explicit bullying
Table 3 Correlations Between Bullying Attitude and Bullying Behavior Measures

${ }^{*} p<0.05, * * p<0.01$

\begin{tabular}{|c|c|c|c|c|c|}
\hline & 1. & 2. & 3. & 4. & 5. \\
\hline \multicolumn{6}{|l|}{ Bulling behavior } \\
\hline \multicolumn{6}{|l|}{ 1. Self-reports } \\
\hline 2. Teacher ratings & $0.43 * *$ & & & & \\
\hline 3. Peer nominations & $0.39 * *$ & $0.64 * *$ & & & \\
\hline \multicolumn{6}{|l|}{ Bullying attitudes } \\
\hline 4. Explicit attitudes & $0.38 * *$ & $0.30^{* *}$ & $0.32 * *$ & & \\
\hline \multicolumn{6}{|l|}{ 5. Implicit attitudes } \\
\hline (General IAT) & 0.07 & 0.02 & -0.06 & -0.08 & \\
\hline \multicolumn{6}{|l|}{ 6. Implicit attitudes } \\
\hline (Specific IAT) & 0.07 & 0.06 & 0.10 & 0.06 & $-0.14^{*}$ \\
\hline
\end{tabular}


Table 4 Summary of General Linear Modeling and Multiple Regression Analysis for Variables Related to Bullying Behavior

\begin{tabular}{|c|c|c|c|c|c|c|}
\hline & \multicolumn{2}{|c|}{ Self-reports } & \multicolumn{2}{|c|}{ Teacher ratings } & \multicolumn{2}{|c|}{ Peer nominations } \\
\hline & $\mathrm{B}$ & SE & $\mathrm{B}$ & SE & $\mathrm{B}$ & $\mathrm{SE}$ \\
\hline Sex & -0.20 & 0.12 & $-0.28 * *$ & 0.08 & $-0.46^{* *}$ & 0.12 \\
\hline Explicit bullying attitudes & $0.34 * *$ & 0.10 & $0.26^{*}$ & 0.10 & $0.39 * *$ & 0.11 \\
\hline Implicit bullying attitudes (General IAT) & 0.11 & 0.07 & 0.00 & 0.14 & -0.15 & 0.16 \\
\hline Implicit bullying attitudes (Specific IAT) & 0.03 & 0.10 & 0.02 & 0.06 & 0.05 & 0.08 \\
\hline Explicit $\times$ implicit bullying attitudes (General IAT) & 0.01 & 0.18 & -0.33 & 0.15 & -0.06 & 0.26 \\
\hline Explicit $\times$ implicit bullying attitudes (Specific IAT) & 0.26 & 0.20 & $0.28 *$ & 0.11 & $0.58 * *$ & 0.09 \\
\hline
\end{tabular}

Bullying (self-reports): $\mathrm{R}^{2}=0.13$; Bullying (teacher-ratings): $\mathrm{R}^{2}=13$; Bullying (peer-nominations): $\mathrm{R}^{2}=0.24 ; * p<0.05 . * * p<0.01$

attitudes. This relation was plotted for three values of explicit bullying attitudes including the mean and the mean plus or minus one standard deviation (Aiken and West 1991). A simple slope analysis (Cohen et al. 2003) showed that only for one of the three slopes, which represents one of the three values of the explicit bullying attitude, implicit bullying attitudes predicted bullying behavior. Implicit bullying attitudes were only related to bullying behavior for children with relatively positive (high scores on) explicit

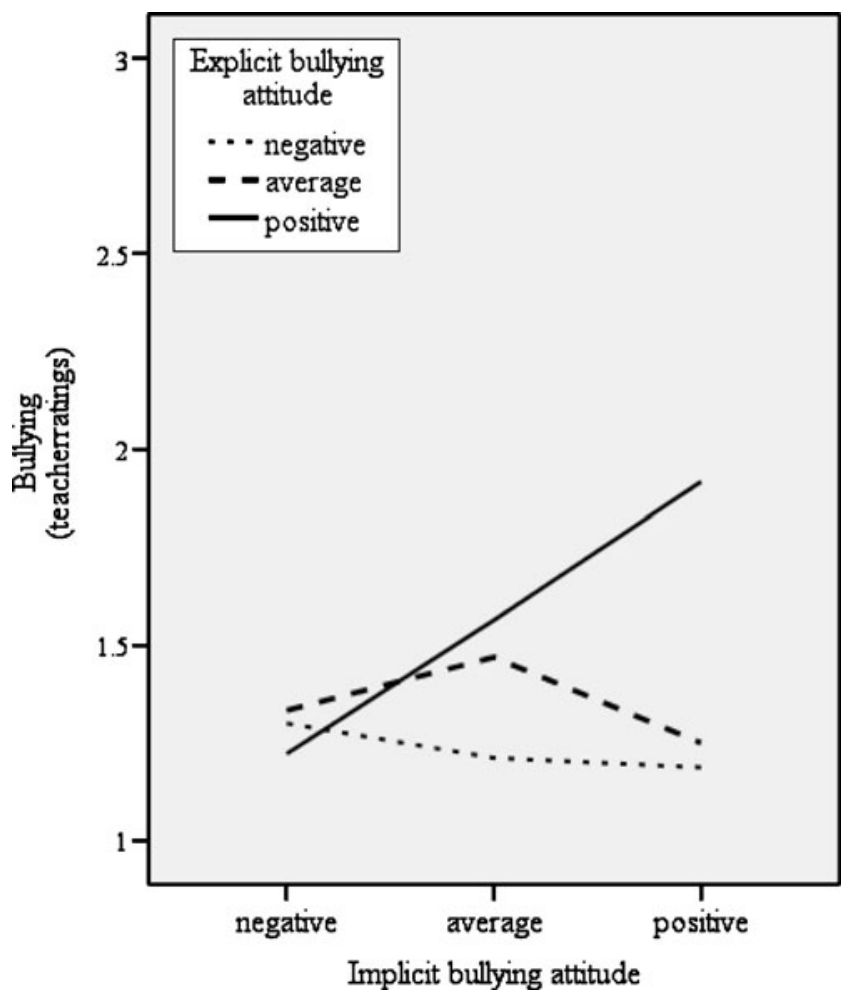

Fig. 1 Interaction between explicit and implicit bullying attitudes in relation to bullying according to teachers. The graph shows the predicted bullying behavior according to teacher ratings (original metric) for participants with negative $(-1 \mathrm{SD})$, average (0 SD), and positive $(+1 \mathrm{SD})$ implicit bullying attitudes (assessed with an IAT) depending on negative ( $-1 \mathrm{SD})$, average (0 SD), and positive (+1 SD) explicit bullying attitudes bullying attitudes ( $+1 \mathrm{SD})$, which is presented as the steep line in the figure. For these children relatively negative (low scores on) implicit bullying attitudes were related to less bullying and relative positive (high scores on) implicit bullying attitudes were related to more bullying $(B=0.62, S E=0.27, p=0.04)$.

For the other values of the explicit bullying attitude the relation between implicit bullying attitudes and bullying was not significant. These non-significant relations are presented as the relatively flat lines in the figure. This means that for children with negative (low scores, - 1 SD) or average explicit bullying attitudes, their implicit bullying attitudes did not seem to affect their bullying behaviors as perceived by their teachers (respectively $B=-0.13, S E=$ $0.23, p=0.57 ; B=-0.04, S E=0.10, p=0.70)$. Figure 2 shows that a similar interaction existed for peer nominations of bullying behavior (for positive explicit bullying attitudes: $B=1.31, S E=0.31, p=0.0001$; for negative explicit bullying attitudes: $B=-0.06, S E=0.25, p=0.81$; for average explicit bullying attitudes: $B=-0.13, S E=0.11, p=0.22$ ). Both for teacher - and peer-rated bullying, the slopes of the three groups of explicit bullying attitudes were also compared. A significant difference was only found for positive explicit bullying attitudes compared to average and negative explicit bullying attitudes; the group of children with positive explicit bullying attitudes scored higher on bullying than the latter two groups, but only when implicit bullying attitudes were positive or average (significant $p$-values ranged from 0 to 0.04 ; for peer-nominations, positive compared to average explicit bullying attitudes did not differ for average implicit bullying attitudes: $p=0.11$ ).

\section{Discussion}

Our study showed that explicit but not implicit bullying attitudes directly predicted bullying behavior. In addition, implicit bullying attitudes interacted with explicit bullying attitudes in the prediction of additional variance in bullying behavior. The interaction indicated that implicit 


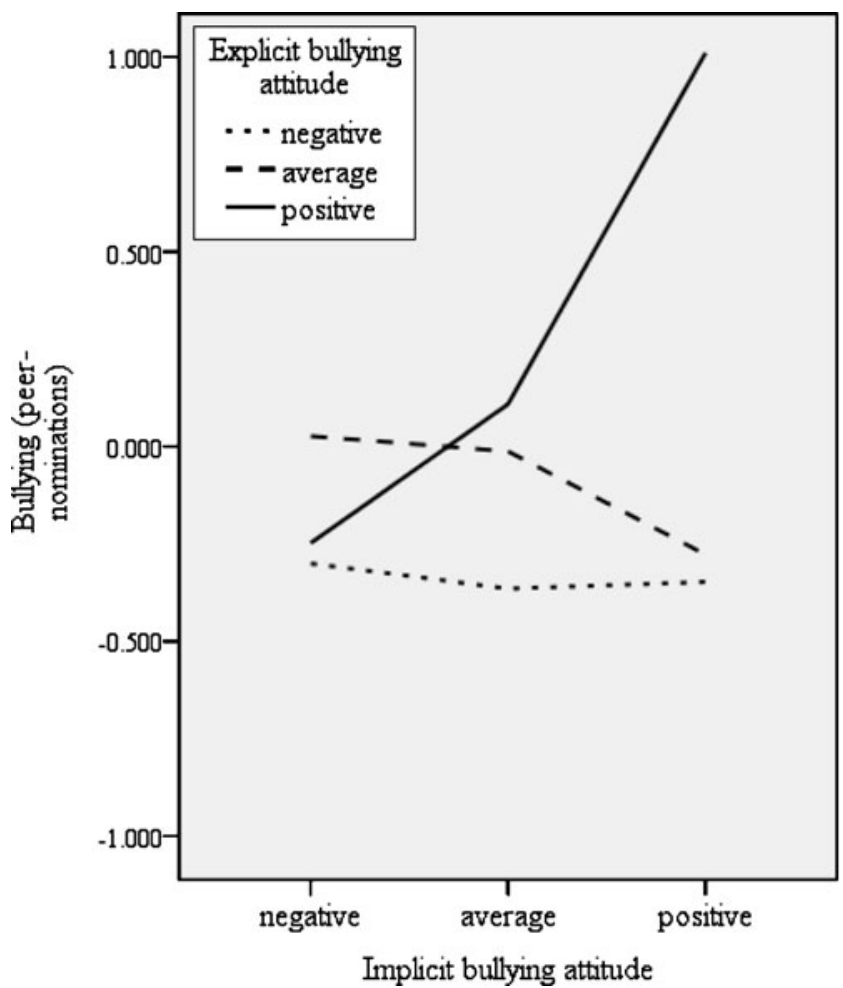

Fig. 2 Interaction between explicit and implicit bullying attitudes in relation to bullying according to peers. The graph shows the predicted bullying behavior according to peer nominations (z-standardized values) for participants with negative $(-1 \mathrm{SD})$, average (0 SD), and positive $(+1 \mathrm{SD})$ implicit bullying attitudes (assessed with an IAT) depending on negative ( $-1 \mathrm{SD})$, average (0 SD), and positive (+1 SD) explicit bullying attitudes

bullying attitudes are important in the prediction of bullying behavior in children who scored relatively positive on explicit bullying attitudes. These findings and their theoretical and practical implications are further discussed.

In line with previous research, we found that most children had relatively negative explicit bullying attitudes (Andreou et al. 2005; Menesini et al. 1997; Salmivalli and Voeten 2004) which corresponds to the anti-bullying policies of schools and bullying programs. In accordance with our expectations, children's explicit bullying attitudes were also related to their bullying behavior. Children who had stronger negative attitudes with regard to bullying, bullied less than children with more positive attitudes towards bullying. Importantly, we found this relation for self-reported bullying as well as peer- and teacher- reported bullying, which extends previous studies that often rely on only one or these two informants.

Secondly, we found that the measures used to assess explicit bullying attitudes and implicit bullying attitudes were not related to each other. This is in accordance with previous research that most often finds only small or nonsignificant relations between implicit- and explicit attitudes (e.g., Hofmann et al. 2005; Perugini and Prestwich 2007).
The finding supports the idea that explicit- and implicit attitudes can not be used to validate one another as they may represent two distinctive kinds of attitudes (e.g., Wilson et al. 2000)

In contrast to our initial expectations, we found that overall, implicit bullying attitudes were not related to bullying behavior. This lack of main effect contrasts with some studies on aggression such as that of Gollwitzer et al. (2007), which did show a relation between their implicit measures and aggression. This could possible be explained by the fact that our study differs from this study in the kind of implicit measures that were used: Gollwitzer et al. (2007) measured the extent to which someone implicitly associated him or herself with aggressive behavior that comprised an individual, behavioral aspect of aggression. In contrast, we measured how positive or negative someone implicitly felt about bullying that comprised a global, affective aspect of aggression. Furthermore, Gollwitzer et al. (2007) focused on implicit aggression that included implicit associations with all kinds of aggressive behavior. Yet, in our study implicit attitudes towards one specific kind of aggressive behavior was measured: bullying, which is defined by the three crucial elements of repetition, harm, and unequal power (Olweus 1999; Rigby 2002).

One explanation for why we did not find a relation between implicit bullying attitudes and bullying behavior could be that implicit bullying attitudes are not related to bullying in general but only to some automatized aspects of bullying. As bullying is viewed as deliberately harming another person, it could be that controlled processes, including explicit bullying attitudes, most strongly influence bullying. However, bullying may also contain aspects that are more automatized (over time). For example, upon seeing the victim an associative network of relevant cognitions could be automatically activated, and then acted upon. These automatic aspects of bullying may be influenced by implicit bullying attitudes. Some indication for this explanation comes from a study of Hofmann et al. (2008), who found that implicit racial attitudes (when they were not inhibited) mainly predicted uncontrolled interracial interactive behaviors such as visual eye contact of the participant and confederate, and the talkativeness of the participant. Explicit racial attitudes, on the other hand, predicted the (explicit) evaluation of the competitiveness of the (interracial) confederate by the participants, which is a controlled aspect of behavior. In future research, it would be interesting to test how implicit bullying attitudes are related to these different aspects of bullying.

Another explanation could be that implicit bullying attitudes are only related to bullying for particular groups of children. Although we did not find a general relation between implicit bullying attitudes and bullying, we did find an interaction between children's implicit and explicit 
bullying attitudes in relation to their bullying behavior when implicit bullying attitudes were measured with the movie-primed IAT. This finding is in accordance with previous studies that found an interaction between explicit and implicit measures when predicting behavior, such as the positivity or negativity of interracial interactions, psychological health and aggression (Hofmann et al. 2008; Schröder-Abé et al. 2007a, 2007b; Wiers et al. 2009). Furthermore we found this interaction when using teacher ratings and peer nominations as indicators of bullying behavior, which increases the validity of the finding. We did not find an interaction between implicit and explicit bullying attitudes for self-reported bullying behavior. Self-reports of bullying may have been influenced by social desirability (even for children with more positive explicit bullying attitudes, social desirability may influence their self-reported bullying behavior, because a child reporting to like bullying may be socially more accepted than a child reporting to be a bully). It can also be the case that some of the children who are identified as bullies by peers or teachers do not perceive their behavior accordingly, because they have some kind of bias in interpreting social behavior (cf. Crick and Dodge 1994). Note that both peer and teacher-reported bullying are often thought of as more objective measures than self reports of bullying (e.g., Blümke and Zumbach 2007; Gollwitzer et al. 2007).

The interaction indicated that implicit bullying attitudes predicted bullying behavior only in children with relatively positive explicit bullying attitudes. When children with relatively positive explicit bullying attitudes also had relatively positive implicit bullying attitudes, they were more likely to bully than children with relatively negative implicit bullying attitudes. In contrast, when children held negative or average explicit bullying attitudes, their implicit attitudes did not predict their bullying behavior. This means that the bullying behavior of these children was only predicted by their explicit bullying attitudes. A possible explanation for this finding could be that for children with negative or average explicit bullying attitudes, the influence of implicit bullying attitudes on bullying behavior is blocked while for children with positive explicit bullying attitudes, implicit bullying attitudes are not blocked and consequently can influence bullying behavior. (Note that the average explicit bullying attitude was very negative).

In accordance with dual process models (e.g., Evans 2008; Gawronski and Bodenhausen 2006; Kahneman 2003; Smith and DeCoster 2000) we propose that the interplay between implicit bullying attitudes, and explicit bullying attitudes predicts bullying behavior. All children have some initial, impulsive, emotional reaction to bullying, i.e. an implicit bullying attitude which could influence their bullying behavior. However, it could be that whether this implicit bullying attitude actually influences this bullying behavior depends on children's explicit bullying attitudes, which is in accordance with the finding that impulsive processes lead to (spontaneous) behavior, if not inhibited by reflective, controlling processes (Sherman et al. 2008). A strong, negative explicit bullying attitude could inhibit and therefore block the influence of (more average or positive) implicit bullying attitudes on bullying behavior, while a positive explicit bullying attitude may not do so. This explanation is based on former studies on inhibition. Inhibition is a mechanism controlling deliberate processes. Former research showed that in the case of high inhibition, explicit attitudes but not implicit attitudes predicted aggression-related behaviors. In the case of low inhibition, implicit attitudes but not explicit attitudes predicted these aggressive behaviors (Hofmann et al. 2008; Wiers et al. 2009). Translated to the present study, it could be that children with a more negative explicit bullying attitude follow a strict anti-bullying rule: thou shall not bully. This explicit anti-bullying attitude could be a strong motivation to inhibit the influence of more initial, impulsive, emotional processes on bullying which may not correspond to the explicit anti-bullying rule (e.g., pro-bullying), also because it is in line with the general notion advocated by schools that bullying should be condemned and will be punished. This inhibiting process could consequently block the influence of implicit bullying attitudes on bullying behavior (flat lines in the Figures).

In contrast, a more positive explicit bullying attitude (note that many children in this category still score below the scale's midpoint), indicates that children have a more lenient explicit attitude towards bullying. For example, these children may have learned that bullying can have both negative consequences such as punishment but also positive consequences such as dominance or social status (e.g., Sijtsema et al. 2009). In this case there is less motivation to inhibit automatically generated, impulsive processes. Consequently, implicit bullying attitudes are not blocked by the explicit bullying attitudes, and therefore they can predict bullying behavior. This is indicated by the steep line in both Figures. Note that for the latter group of children who have pro-bullying attitudes on an explicit level, only those who also hold positive implicit bullying attitudes are involved in bullying. Those who hold negative implicit attitudes are less or not involved in bullying. In that sense there may be some kind of threshold in terms of positive attitudes (both implicit and explicit bullying attitudes are positive) that needs to be reached before children start bullying. Note that this conclusion should be considered as tentative, given that this is the first study to examine implicit bullying attitudes in addition to explicit bullying attitudes.

Our findings suggest that implicit bullying attitudes could play an even more important role in determining the bullying behavior of these children when these attitudes are 
further activated with a prime, which in our study was a movie on bullying. This could also help to explain why we found a significant interaction using the movie-primed IAT but no interaction using the general IAT. It could be that the relevant associations on bullying were more strongly activated after seeing a movie than when no prior movie was presented. Consequently, children's implicit bullying attitudes may have been better represented in the movie-primed IAT than in the general IAT that was not preceded by a movie-prime. This in accordance with former research who found that implicit attitudes predicted behavior (only) after using a prime (Perugini and Prestwich 2007; Rustad et al. 2003; Schoenmakers et al. 2008), or in a certain context functioning as a prime (e.g., a hospital environment was suggested to have different effects on food-related associations than a neutral environment; Roefs et al. 2005).

Furthermore, in line with our explanation of this interaction, it could also be important to include social or contextual factors in future bullying-attitude research as children's bullying attitudes and behavior can be influenced by the bullying norms or rules children learn and the bullying experiences children have in their social environments including their family environment, and particularly their peer environment (e.g., Pintado 2007; Salmivalli and Voeten 2004). For example, research has shown that bullying that is normative in the peer group or is rewarded by status, popularity, or acceptance in this group might trigger more favourable attitudes toward bullying (e.g., Sijtsema et al. 2009). Therefore it could be interesting to explore whether the implicit- and explicit bullying attitudes of children can be predicted by the bullying behavior and attitudes of their peers (in the classroom). In addition, it would also be useful to study bullying attitudes in relation to other bully- and victim-related behaviors such as being victimization, being a bystander, or non-involved (Salmivalli and Voeten 2004).

Although this study generated interesting results, a number of limitations should be acknowledged. First of all, instead of a fixed administration order to assess explicit - and implicit bullying attitudes it would have been better to have randomized this administration order to prevent any order effects.

Second, we found that these IATs were negatively related to each other which suggests that these two versions possibly measured somewhat different concepts. However, in IAT research it is rare to find substantial (positive) correlations between different implicit measures designed to assess the same concept. For example, Bosson et al. (2000) assessed measures of implicit self-esteem and found very low and (often) non-significant correlations. This is often explained by the fact that even subtle changes in a task can trigger different associative processes (e.g. Mitchell et al. 2003), which result in attitudes that contain various aspects of the concept which is studied. During the past decade there has been a great deal of research investigating the validity of the IAT in predicting behavior, including research into moderating the effects (e.g. Hofmann et al. 2005) and research into the influence of different varieties of the IAT with respect to the outcomes measured. For example, it has been found that a slightly changed "personalized" IAT assessed in somewhat different associations than the "standard" IAT (Houben and Wiers 2007; Nosek and Hansen 2008; Olson and Fazio 2004). By implication, it is likely that the IAT which used pictures from the bullying movie (i.e., the pictures are likely to have acquired personal emotional meaning due to the movie) assessed something different from the general IAT which assesses associations with the general concept of bullying (e.g. cultural knowledge that is available in memory which is not necessary the way a person feels about the attitude object him or herself; Olson and Fazio 2004).

Accordingly, our measures of the IAT could have tapped into different aspects of implicit bullying attitudes. However, because this study is the first to use these measures these explanations may be preliminary. Therefore both measures have to be further studied and validated. For example, results on bullying behavior in relation to an IAT containing other bullying pictures could be compared with the results of our study to examine the convergent validity and the predictive validity of the bullying IAT. To test whether a prime is necessary to activate implicit bullying attitudes, two conditions of this IAT could be compared: the IAT preceded by a movie prime and the same IAT without the movie. Furthermore, implicit bullying attitudes could be measured with other implicit measures such as a priming measure (a priming measure refers to a task in which the response time to target information following a prime indicates the strength of the association between prime and target; e.g., O'Connor et al. 2007).

In addition, the correlational design of our study ultimately does not allow for making conclusions about any causal links between bullying attitudes and bullying behavior. Bullying-related attitudes may influence children's bullying but it can also be that over time, a child adopts beliefs on bullying that are consistent with his or her bullying behavior, which is a well known mechanism applied when persons experience a certain cognitive dissonance (Festinger 1957). Although there is some evidence that for children at the end of primary school, individual beliefs about aggression influence aggressive behavior rather than the other way around (Huesmann and Guerra 1997), the causal directions between bullying attitudes and bullying should be further studied.

The present study was, as far as we know, the first to examine implicit bullying attitudes in relation to bullying behavior and used multiple sources for assessing bullying 
involvement (self reports, teacher ratings and peer nominations). Furthermore, our study suggests that there are individual differences in the relative strength of cognitive processes underlying bullying. In children with negative explicit bullying attitudes, only these (negative) explicit attitudes about bullying but not their implicit bullying attitudes were found to be related to their bullying behavior. In children without clear anti-bullying explicit attitudes, implicit bullying attitudes were found to be related to their bullying behavior. This suggests that to be able to predict the bullying behavior of all groups of children better, not only explicit bullying attitudes should be considered but also implicit bullying attitudes, and the combination of explicit - and implicit bullying attitudes.

Finally, our findings may have some practical implications. They indicate that a primed IAT may be a valid measure to assess implicit bullying attitudes. In the future, it could possibly be a suitable instrument to use in schools because data can be obtained relatively quickly and easily. Even more, IATs can also be administered and processed through the internet which makes it an even more attractive instrument to use (Nosek 2005). Furthermore, our findings may also have some implications for anti-bullying programs. First, they seem to underscore the importance of focusing on explicit bullying attitudes and teaching clear anti-bullying rules, as negative explicit bullying attitudes are related to little or less bullying behavior and appear to protect against the influence of pro-bullying attitudes on an implicit level. Relying on dual process models, explicit bullying attitudes may be tackled best by including techniques that rely on thought and conscious reasoning such as persuasion or increasing awareness of bullying behavior, which are the techniques that are most frequently used in current anti-bullying programs (e.g., Smith et al. 2004; Vreeman and Carroll 2007). Second, they suggest that especially for children with relatively positive explicit bullying attitudes whose bullying behavior is influenced by their implicit bullying attitudes, current anti-bullying programs may be improved by also including techniques that rely on emotion (Dasgupta and Greenwald 2001; Edwards 1990; Edwards and von Hippel 1995; Fabrigar and Petty 1999) and automatism such as establishing associations between bullying and negative feelings and experiences. In conclusion, this first study on the impact of implicit attitudes in the prediction of bullying suggests that implicit bullying attitudes predict bullying in a subgroup of children who have relatively positive explicit bullying attitudes.

Acknowledgements We want to thank Daan Creemers for his assistance with the data collection, Rinus Voeten for his assistance with data analysis, and Giovanni ten Brink for his assistance with technical realization of the IAT.

\section{References}

Aiken, L. S., \& West, S. G. (1991). Multiple regression: Testing and interpreting interactions. Thousand Oaks: Sage Publications, Inc.

Andreou, E., Vlachoub, A., \& Didaskaloub, E. (2005). The roles of self-efficacy, peer interactions and attitudes in bully-victim incidents. Implications for intervention policy-practices. School Psychology International, 26, 545-562.

Asendorpf, J. B., Banse, R., \& Mucke, D. (2002). Double dissociation between implicit and explicit personality self-concept: The case of shy behavior. Journal of Personality and Social Psychology, 83, 380-393.

Baron, A. S., \& Banaji, M. R. (2006). The development of implicit attitudes: Evidence of race evaluations from ages 6 and 10 and adulthood. Psychological Science, 17, 53-58.

Berger, K. S. (2007). Update on bullying at school: Science forgotten? Developmental Review, 27, 90-126.

Blümke, M., \& Zumbach, J. (2007). Implicit and explicit measures in analyzing aggression of computer gamers. In G. Steffgen \& M. Gollwitzer (Eds.), Emotions and aggressive behavior (pp. 3756). Göttingen: Hogrefe \& Huber.

Bosson, J. K., Swarm, W. B., \& Pennebaker, J. W. (2000). Stalking the perfect measure of implicit self-esteem: The blind men and the elephant revisited? Journal of Personality and Social Psychology, 79, 631-643.

Boulton, M. J., Trueman, M., \& Flemington, I. (2002). Associations between secondary school pupils' definitions of bullying, attitudes towards bullying, and tendencies to engage in bullying: Age and sex differences. Educational Studies, 28, 352-370.

Cohen, J., Cohen, P., West, S. G., \& Aiken, L. S. (2003). Applied multiple regression. Correlation analysis for the behavioral sciences, 3rd edn. Lawrence Erlbaum Associates, Lawrence Erlbaum Associates.

Crick, N. R., \& Dodge, K. A. (1994). A review and reformulation of social information- processing mechanisms in children's social adjustment. Psychological Bulletin, 115, 74-101.

Dasgupta, N., \& Greenwald, A. G. (2001). On the malleability of automatic attitudes: Combating automatic prejudice with images of admired and disliked individuals. Journal of Personality and Social Psychology, 81, 800-814.

Edwards, K. (1990). The interplay of affect and cognition in attitude formation and change. Journal of Personality and Social Psychology, 59, 200-216.

Edwards, K., \& von Hippel, W. (1995). Hearts and minds: The priority of affective versus cognitive factors in person perception. Personality and Social Psychology Bulletin, 21, 996-1011.

Evans, J. S. B. T. (2008). Dual-processing accounts of reasoning, judgment, and social cognition. Annual Review of Psychology, 59, 255-278.

Fabrigar, L. R., \& Petty, R. E. (1999). The role of the affective and cognitive bases of attitudes in susceptibility to affectively and cognitively based persuasion. Personality and Social Psychology Bulletin, 25, 363-381.

Festinger, L. (1957). A theory of cognitive dissonance. Evanston: Row, Peterson.

Gawronski, B., \& Bodenhausen, G. V. (2006). Associative and propositional in evaluation: An integrative review of implicit and explicit attitude change. Psychological Bulletin, 132, 692731.

Glasman, L. R., \& Albarracin, D. (2006). Forming attitudes that predict future behavior: A meta-analysis of the attitude-behavior relation. Psychology Bulletin, 132, 778-822.

Gollwitzer, M., Banse, R., Eisenbach, K., \& Naumann, A. (2007). Effectiveness of the Vienna Social Competence Training on 
explicit and implicit aggression evidence from an aggressivenessIAT. European Journal of Psychological Assessment, 23, 150 156

Greenwald, A. G., McGhee, D. E., \& Schwartz, J. L. K. (1998). Measuring individual differences in implicit cognition: The implicit association test. Journal of Personality and Social Psychology, 74, 1464-1480.

Greenwald, A. G., Nosek, B. A., \& Banaji, M. R. (2003). Understanding and using the implicit association test: I. An improved scoring algorithm. Journal of Personality and Social Psychology, 85, 197-216.

Greenwald, A. G., Nosek, B. A., \& Siriam, N. (2006). Consequential validity of the implicit association test: Comment on Blanton and Jaccard. American Psychologist, 61, 56-61.

Grenard, J. L., Ames, S. L., Wiers, R. W., Thush, C., Sussman, S., \& Stacy, A. W. (2008). Working memory capacity moderates the predictive effects of drug-related assocations on substance use. Psychology of Addictive Behaviors, 22, 426-432.

Hofmann, W., Gawronski, B., Gschwendner, T., Le, H., \& Schmitt, M. (2005). A meta-analysis on the correlation between the implicit association test and explicit self-report measures. Personality and Social Psychology Bulletin, 31, 1369-1385.

Hofmann, W., Gschwendner, T., Castelli, L., \& Schmitt, M. (2008). Implicit and explicit attitudes and interracial interaction: The moderating role of situationally available control resources. Group Processes \& Intergroup Relations, 11, 69-87.

Houben, K., \& Wiers, R. W. (2007). Personalizing the alcohol-IAT with individualized stimuli: Relationship with drinking behaviour and drinking-related problems. Addictive Behaviors, 32, 2852 2864.

Huesmann, L. R., \& Guerra, N. G. (1997). Children's normative beliefs about aggression and aggressive behavior. Journal of Personality and Social Psychology, 72, 408-419.

Kahneman, D. (2003). A perspective on judgment and choice: Mapping bounded rationality. American Psychologist, 58, 697720.

Menesini, E., Eslea, M., Smith, P. K., Genta, M. L., Giannetti, E., Fonzi, A., et al. (1997). Cross-national comparison of children's attitudes towards bully/victim problems in school. Aggressive Behavior, 23, 245-257.

Menesini, E., Codecasa, E., Benelli, B., \& Cowie, H. (2003). Enhancing children's responsibility to take action against bullying: Evaluation of a befriending intervention in Italian middle schools. Aggressive Behavior, 29, 1-14.

Mitchell, J. P., Nosek, B. A., \& Banaji, M. R. (2003). Contextual variations in implicit evaluation. Journal of Experimental Psychology: General, 132, 455-469.

Newcomb, A. F., \& Bukowski, W. M. (1983). Social impact and social preference as determinants of children's peer group status. Developmental Psychology, 19, 856-867.

Nosek, B. A. (2005). Moderators of the relationship between implicit and explicit evaluation. Journal of Experimental Psychology, $134,565-584$

Nosek, B. A., Greenwald, A. G., \& Banaji, M. R. (2005). Understanding and using the implicit association test: II. Method variables and construct validity. Personality and Social Psychology Bulletin, 31, 166-180.

Nosek, B. A., \& Hansen, J. J. (2008). Personalizing the implicit association test increases explicit evaluation of target concepts. European Journal of Psychological Assessment, 24, 226236.

O’Connor, R. M., Fite, P. J., Nowlin, P. R., \& Colder, C. R. (2007). Children's beliefs about substance use: An examination of age differences in implicit and explicit cognitive precursors of substance use initiation. Psychology of Addictive Behaviors, 21, $525-533$.
Olson, M. A., \& Fazio, R. H. (2004). Reducing the influence of extrapersonal associations on the implicit association test: Personalizing the IAT. Journal of Personality and Social Psychology, 86, 653-667.

Olweus, D. (1989). The Olweus Bully/victim questionnaire. Bergen: Mimeo.

Olweus, D. (1993). Bullying at school: What we know and what we can do. Oxford: Blackwell.

Olweus, D. (1999). In P. K. Smith, Y. Morita, J. Junger-Tas, D. Olweus, R. Catalano, P. Slee (eds.), The nature of school bullying: A crossnational perspective (pp. 7-27). New York: Routledge.

Perugini, M., \& Prestwich, A. (2007). The gatekeeper: Individual differences are key in the chain from perception to behavior. European Journal of Personality, 21, 303-317.

Petty, R., \& Cacioppo, J. (1986). Communication and persuasion: Central and peripheral routes to attitude change. New York: Springer-Verlag.

Pintado, I. (2007). Perceptions of school climate and bullying in middle schools. Dissertation-Abstracts-International: Section B: The Sciences and Engineering, 68, 244.

Rigby, K. (2002). New perspectives on bullying. London: Jessica Kingsley.

Rigby, K. (2004). Addressing bullying in schools. Theoretical perspectives and their implications. School Psychology International, 25, 287-300.

Roefs, A., Stapert, D., Isabella, L. A. S., Wolters, G., Wojciechowski, F., \& Jansen, A. (2005). Early associations with food in anorexia nervosa patients and obese people assessed in the affective priming paradigm. Eating Behaviors, 6, 151-163.

Rustad, R. A., Small, J. E., Jobes, D. A., Safer, M. A., \& Peterson, R. J. (2003). The impact of rock videos and music with suicidal content on thoughts and attitudes about suicide. Suicide and Lifethreatening Behavior, 33, 120-131.

Salmivalli, C., \& Voeten, M. (2004). Connections between attitudes, group norms, and behaviour in bullying situations. International Journal of Behavioral Development, 28, 246-258.

Schoenmakers, T., Wiers, R. W., \& Field, M. (2008). Effects of a low dose of alcohol on cognitive biases and craving in heavy drinkers. Psychopharmacology, 197, 169-178.

Scholte, R. H. J., de Kemp, R. A. T., Haselager, G. J. M., \& Engels, R. C. M. E. (2007). Longitudinal stability in bullying and victimization in childhood and adolescence. Journal of Abnormal Child Psychology, 35, 217-238.

Schröder-Abé, M., Rudolph, A., Wiesner, A., \& Schütz, A. (2007a). High implicit self-esteem is not necessarily advantageous: Discrepancies between explicit and implicit self-esteem and their relationship with anger expression and psychological health. European Journal of Personality, 21, 319-339.

Schröder-Abé, M., Rudolph, A., Wiesner, A., \& Schütz, A. (2007b). Self-esteem discrepancies and defensive reactions to social feedback. International Journal of Psychology, 42, 174-183.

Sherman, J. W., Gawronski, B., Gonsalkorale, K., Hugenberg, K., Allen, T. J., \& Groom, C. J. (2008). The self-regulation of automatic associations and behavioral impulses. Psychology Review, 115, 314-335.

Sijtsema, J. J., Veenstra, R., Lindenberg, S., \& Salmivalli, C. (2009). Empirical test of bullies' status goals: Assessing direct goals, aggression, and prestige. Aggressive Behavior, 35, 57-67.

Smith, E., \& DeCoster, J. (2000). Dual-process models in social and cognitive psychology: Conceptual integration and links to underlying memory systems. Personality and Social Psychology Review, 4, 108-131.

Smith, J. D., Schneider, B. H., Smith, P. K., \& Ananiadou, K. (2004). The effectiveness of whole-school anti-bullying programs: A synthesis of evaluation research. School Psychology Review, 33, $547-560$. 
Solberg, M. E., \& Olweus, D. (2003). Prevalence estimation of school bullying with the Olweus Bully/Victim Questionnaire. Aggressive Behavior, 29, 239-268.

Stevens, V., Van Oost, P., \& De Bourdeaudhuij, I. (2000). The effects of an anti-bullying intervention programmes on peers' attitudes and behaviour. Journal of Adolescence, 23, 21-34.

Sullivan, K. (2000). The anti-bullying handbook. Oxford: OUP.

Thissen, M., \& Bendermacher, N. (1996). Kunst SocStat: Sociometric status. Nijmegen: University of Nijmegen.

Thush, C., Wiers, R. W., Ames, S. L., Grenard, J. L., Sussman, S., \& Stacy, A. W. (2008). Interactions between implicit and explicit cognition and working memory capacity in the prediction of alcohol use in at-risk adolescents. Drug and Alcohol Dependence, 94, 116-124.

Vreeman, R. C., \& Carroll, A. E. (2007). A systematic review of school-based interventions to prevent bullying. Archives of Pediatrics \& Adolescent Medicine, 161, 78-88.

Wiers, R. W., Beckers, L., Houben, K., \& Hofmann, W. (2009). A short fuse after alcohol: Implicit power associations predict aggressiveness after alcohol consumption in young heavy drinkers with limited executive control. Pharmacology, Biochemistry and Behavior, 93, 300-305.

Wilson, T. D., Lindsey, S., \& Schooler, T. Y. (2000). A model of dual attitudes. Psychological Review, 107, 101-126. 